IP Periodica Polytechnica Electrical Engineering and Computer Science

59(2), pp. 43-47, 2015

DOI: $10.3311 /$ PPee. 8202

Creative Commons Attribution (i)

RESEARCH ARTICLE

\section{Approximate and Proper Electromagnetic Modelling in Moving Conductors}

\author{
Sándor Bilicz ${ }^{1 *}$
}

Received 04 May 2015; accepted after revision 25 June 2015

\begin{abstract}
A conductor moving in a stationary magnetic field often rises crucial issues at the courses on electromagnetics for electrical engineering students. The correct use of Faraday's induction law can sometimes be harder than one would think for the first sight. In this paper, we revisit two simple examples of eddycurrents by means of numerical field computation. First, the case of a small magnet falling within a copper tube -which is an impressive experiment as well-is dealt with. Second, a metallic plate moving below a small magnet is examined. In both cases, approximate and proper electromagnetic models are compared. The approximate solutions are usually of satisfying accuracy, but they hide some parts of the physics behind the phenomenon. At the university courses, however, the deep understanding of the electromagnetics must precede the use of practical simplifications, even when using an up-to-date numerical field computation software.
\end{abstract}

\section{Keywords}

moving conductor, electromagnetic induction, finite elements

\footnotetext{
${ }^{1}$ Department of Broadband Infocommunications and Electromagnetic Theory, Budapest University of Technology and Economics

H-1521 Budapest, P.O.B. 91., Hungary
}

*Corresponding author, e-mail: bilicz@evt.bme.hu

\section{Introduction}

One of the most impressive demonstrations of the eddy-currents is the damped fall of a strong magnet in a non-ferromagnetic conducting tube. The magnet's steady state velocity is much smaller than in free-space due to the braking effect of the induced eddy-currents within the tube wall. This experiment is perfect for focusing the young students' interest on electromagnetic phenomena and also for teaching quantitative modelling for graduate students.

Several analytical (e.g., [1-4]) and experimental (e.g., [5] and [4] again) approaches have recently been published on this demonstration example. A common concern about these works is that they consider the magnetic field generated by the falling magnet only and neglect the magnetic field risen due to the currents induced within the tube wall. This second part of the induction is much smaller than the first one in the standard configurations at relatively small falling velocities, thus, its neglection is reasonable from a numerical point of view.

The assumption of "small" speed -as a condition for the neglection of the reaction field- also occurs in other common examples, like the metal plate moving below a magnet $[6,7]$. Within the frame of the Lorentz force velocimetry [8], or Lorentz force eddy-current testing [9], again a similar approximation is usually made, that is referred to as "weak reaction approach".

However, from the viewpoint of the education, the attempt to model the whole phenomenon might sometimes be more useful than a good approximation (which can easily be misunderstood by the students). A common mistake in students' thinking is to force sequential rules even if there is no distinguished order of the phenomena but they all interact with each other [10]. Our examples with a "reaction-effect" might help the students to see the electromagnetics more clearly.

Hereafter in this paper, we call a model "proper" if it takes into account the reaction magnetic field, and we use the term "approximate" when referring to a model which neglects the reaction effect.

We present the EM modelling and the 2-dimensional Finite Element Method (FEM) simulation of the magnet falling in a conductive tube and of the metal plate moving below a magnet. 
We study the relation between the results obtained by the approximate and the proper models, with respect to the velocity of the movement.

\section{Small magnet falling in a tube}

\subsection{The studied configuration and the EM model}

Let us consider a very long tube in which the magnet falls with a constant velocity $\mathbf{v}$ (the sum of all forces acting on the magnet -gravity, drag and magnetic-gives zero). Herein $\mathbf{v}$ is assumed to be known. The magnet is assumed to be small, i.e., it is modelled by a magnetic dipole with a vertical moment, moving on the axis of the tube $(z)$, see Fig. 1.

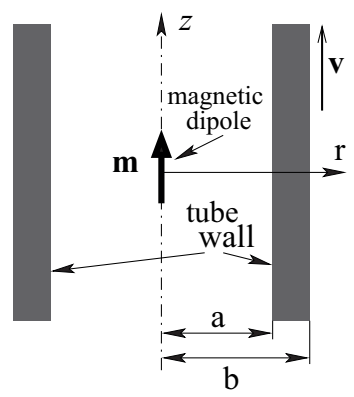

Fig. 1 The axisymmetric configuration in a cylindrical coordintate system $(z, r$, $\varphi$ ). A magnetic dipole (with a $z$-directed moment $\mathbf{m}$ ) is at rest on the axis of an infinite-long tube which moves with a velocity $\mathbf{v}$.

Since only the relative motion of the magnet and the tube counts, in our model, we fix the magnet to the center of the cylindrical coordinate system and the tube is assumed to move to the $+z$ direction with a velocity $\mathbf{v}=\hat{\mathbf{e}} v_{z}$. Let us denote the magnetic induction of the dipole by $\mathbf{B}_{0}$ (expression is available in textbooks, e.g., in [11]). Our goal is to obtain the current density $\mathbf{J}$ within the tube wall.

The constitutive relation in the moving conductor ${ }^{1}$-as a consequence of Faraday's induction law is:

$$
\mathbf{J}=\sigma(\mathbf{E}+\mathbf{v} \times \mathbf{B}),
$$

where $\mathbf{E}$ and $\mathbf{B}$ are the electric field and the magnetic induction in the rest-frame, respectively. $\mathbf{E}$ is zero, since no static charge is experienced anywhere in the conductor. This is explained by the axial symmetry of the configuration and by the fact that $\mathbf{J}$ has an azimuthal component only. Equation (1) includes B, which is the total magnetic induction in the rest-frame.

As a first approach, let us assume $\mathbf{B}=\mathbf{B}_{0}$, i.e., let us neglect the induction associated with the current in the tube wall. In so doing, the current density -now denoted by $\mathbf{J}_{0}$ - can easily be expressed:

$$
\mathbf{J}_{0}=\sigma \mathbf{v} \times \mathbf{B}_{0}=\hat{\mathbf{e}}_{\varphi} \sigma v_{Z} B_{0 r}
$$

where $B_{0 r}$ is the radial component of the induction of the dipole.

1 For the sake of rigour: the velocity has to be much smaller than the speed of light.
In the proper model, however, we have to write the total induction as a sum of two terms, $\mathbf{B}=\mathbf{B}_{0}+\mathbf{B}_{e}$, where the so far neglected second term is generated by the currents in the tube wall. Let us derive $\mathbf{B}_{e}$ from a vector potential: $\mathbf{B}_{e}=\nabla \times \mathbf{A}$. This potential satisfies the Poisson's equation (with the gauge $\nabla \cdot \mathbf{A}=0$ ):

$$
\Delta \mathbf{A}=-\mu_{0} \mathbf{J} .
$$

Rewriting this into (1), we get:

$$
-\Delta \mathbf{A}-\mu_{0} \sigma \mathbf{v} \times(\nabla \times \mathbf{A})=\mu_{0} \sigma\left(\mathbf{v} \times \mathbf{B}_{0}\right) .
$$

Since $\mathbf{B}_{e}$ has axial $(z)$ and radial $(r)$ components only, $\mathbf{A}$ is azimuthal: $\mathbf{A}=A_{\varphi}(z, r) \hat{\mathrm{e}}_{\varphi}$. The differential equation for $A_{\varphi}$ is:

$$
-\frac{\partial}{\partial r}\left(r \frac{\partial A_{\varphi}}{\partial r}\right)-\frac{\partial}{\partial z}\left(r \frac{\partial A_{\varphi}}{\partial z}\right)+\frac{A_{\varphi}}{r}+r \mu_{0} \sigma v_{z} \frac{\partial A_{\varphi}}{\partial z}=r \mu_{0} \sigma v_{z} B_{0 r} .
$$

In the air-filled regions inside and outside the tube, $\sigma=0$ is set in (5). $A_{\varphi}$ is continuous at the boundaries and vanishes at infinity.

Equation (5) cannot be analytically solved; we use the Finite Element Method. In the PDE-toolbox of Matlab ${ }^{\circledR}$ the elliptic equation scheme is used, and the term containing $\frac{\partial A_{\varphi}}{\partial z}$ is put to the right side and the equation is solved as a nonlinear one. Once $A_{\varphi}$ is obtained, the current density is given by (3).

The obtained current density distribution makes the calculation of the force acting on the magnet possible. This can be used for, e.g., the calculation of the steady state velocity of the fall, when the mass of the magnet is known and the drag forces are neglected (this is out of the scope of this paper).

\subsection{Numerical example for the magnet falling in a tube}

The studied tube is made of copper (conductivity: $\sigma=57$ $\mathrm{MS} / \mathrm{m})$. Let its inner and outer radii be $a=7.85 \mathrm{~mm}$ and $b=$ $9.75 \mathrm{~mm}$, respectively. The magnetic moment $\mathbf{m}$ of the dipole which models the small magnet is not given, we only know that $\mathbf{m}$ is $z$-directed. Let us note that the solution of (5) linearly depends on $\mathrm{B}_{0 r}$, and so on $\mathbf{m}$ as well. That is, the magnitude of $\mathbf{m}$ can be arbitrarily chosen and we are free to study the normalised current density only.

The current densities at the inner tube wall, along $z$, have been calculated and plotted for two velocities $(2 \mathrm{~m} / \mathrm{s}$ and $10 \mathrm{~m} / \mathrm{s}$ ) in Fig. 2. The discrepancy between the results of the approximate and the proper model gets larger as the velocity increases, as expected. The typical velocities in such experiments are smaller than about $2 \mathrm{~m} / \mathrm{s}$ - which corresponds to the first case presented in Fig. 2 -, thus, the approximate model provides satisfying accuracy. However, the limitation of the approximation has been pointed out. At a velocity of $10 \mathrm{~m} / \mathrm{s}$, a significant difference is experienced between the results of the approximate and proper models. The current distribution is not symmetric to the origin, in contrast with the prediction of the approximate model. 

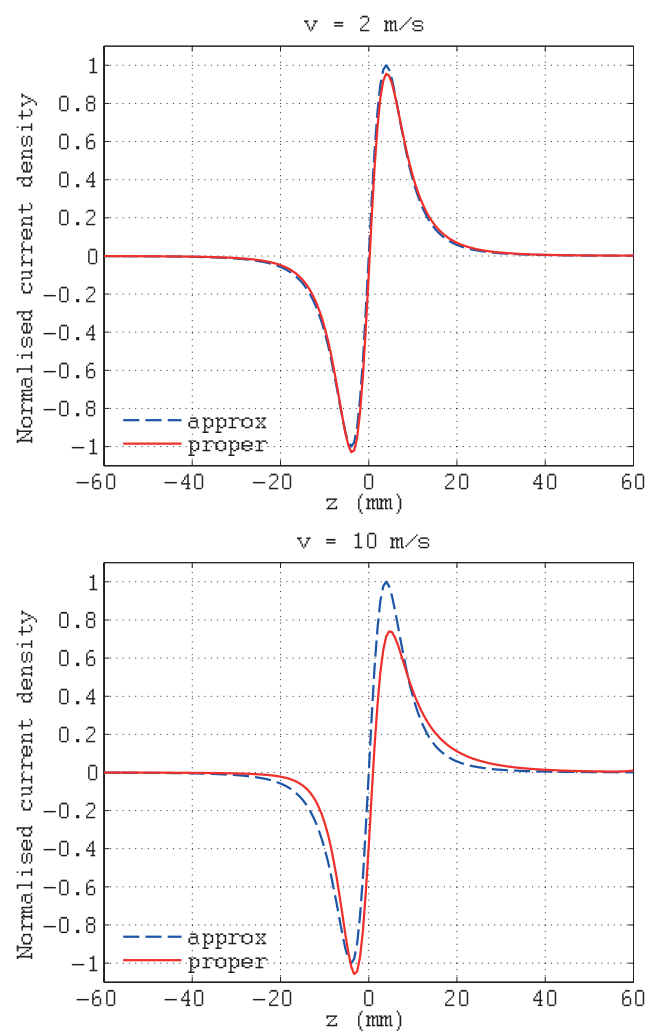

Fig. 2 Tube-example: normalised current densities on the inner wall of the tube at two different falling velocities.

\section{A plate moving below a magnetic dipole 3.1 Configuration and EM model}

As a second example, an infinite, non-magnetic, conducting plate of thickness $a$ is considered, which moves with a constant velocity $\mathbf{v}=v_{x} \hat{\mathrm{e}}_{x}$ (Cartesian coordinates are used in this case), as sketched in Fig. 3. The plate surfaces are the $z= \pm a / 2$ planes. A $z$-directed magnetic dipole is placed to the point $(0,0, h)$, where $h \gg a$ holds. Due to the relative motion of the conductor and the magnetic field of the dipole, an electromotive force is induced and it drives a current within the plate. As the plate is thin, the $z$ component of the current density $\mathbf{J}$ is neglected, moreover, $\mathbf{J}$ is assumed to be constant along $z$. When considering the interaction between the dipole field and the current within the plate, these assumptions on $\mathbf{J}$ enable us to model the current distribution by a surface current $\mathbf{K}$ within the plane $z=0$ :

$$
\mathbf{K}_{(x, y)}:=\int_{-a / 2}^{a / 2} \mathbf{J}(x, y, z) \mathrm{d} z, \quad \hat{\mathbf{e}}_{z} \cdot \mathbf{J}(x, y, z) \equiv 0 .
$$

The constitutive relation (1) now gives

$$
\mathbf{K}=a \sigma(\mathbf{E}+\mathbf{v} \times \mathbf{B}) .
$$

In contrary with the tube-case, the electric field in the rest frame $\mathbf{E}$ is not zero, because of the static charge distribution arising within the conductor [6,7]. As we consider the steady state, the electric field can be derived from a scalar potential. The components of $\mathbf{E}$ within the $x y$ plane are expressed as $\mathbf{E}$ $=-\nabla_{t} \phi$, where $\nabla_{t}$ is the tangential gradient in the $x y$ plane.

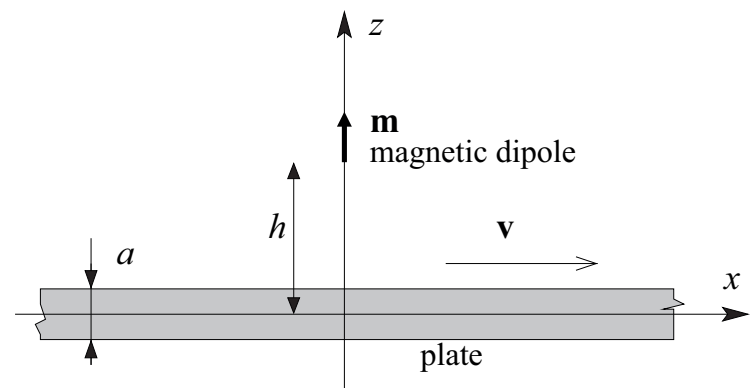

Fig. 3 A magnetic dipole (with a $z$-directed moment $\mathbf{m}$ ) is at rest above the infinite plate of thickness $a$. The plate moves to the $\mathrm{x}$ direction with a velocity $\mathbf{v}$.

The magnetic induction has two terms again: the incident field generated by the dipole and the "reaction field" due to the induced current within the plate: $\mathbf{B}=\mathbf{B}_{0}+\mathbf{B}_{e}$. The dipole field $\mathbf{B}_{0}$ has an analytical expression; whereas the reaction field can be expressed by means of the Biot-Savart law, knowing the surface current density $\mathbf{K}$. Let the operator $\mathcal{B}$ denote the application of the Biot-Savart law as follows:

$$
\begin{aligned}
\mathbf{B}_{e}(x, y) & =\hat{\mathbf{e}}_{z} B_{e z}(x, y)=\mathcal{B}\{\mathbf{K}(x, y)\} \\
& =\frac{\mu_{0}}{4 \pi} \int_{S} \frac{\boldsymbol{K}\left(x^{\prime}, y^{\prime}\right) \times\left[\left(x-x^{\prime}\right) \hat{\mathbf{e}}_{x}+\left(y-y^{\prime}\right) \hat{\mathbf{e}}_{y}\right]}{\left[\left(x-x^{\prime}\right)^{2}+\left(y-y^{\prime}\right)^{2}\right]^{3 / 2}} \mathrm{~d} S^{\prime},
\end{aligned}
$$

where $S$ stands for the whole $x y$ plane.

The continuity equation implies that $\mathbf{K}$ is source-free, i.e. (7) leads to

$$
0=\nabla \cdot\left(-\nabla_{t} \phi+\mathbf{v} \times \hat{\mathbf{e}}_{z}\left(B_{0 z}+B_{e z}\right)\right) .
$$

Let us note that $\mathbf{B}_{0}$ does have $x$ and $y$ components as well in the $x y$ plane, but neither of them causes electromotive force tangential to the $x y$ plane.

As a first "approximate" approach -most commonly used in the literature-, the reaction field $\mathbf{B}_{e}$ is neglected and simply the Poisson's equation

$$
-\nabla \cdot \nabla_{t} \phi=-\nabla \cdot\left(\mathbf{v} \times \hat{\mathbf{e}}_{z} B_{0 z}\right)=v_{x} \frac{\partial B_{0 z}}{\partial y}
$$

is solved for $\phi$.

Our proper model takes into account $\mathbf{B}$, and expresses it by using (8) as:

$$
\hat{\mathbf{e}}_{z} B_{e z}=a \sigma \mathcal{B}\left\{-\nabla_{t} \phi+\mathbf{v} \times \hat{\mathbf{e}}_{z}\left(B_{0 z}+B_{e z}\right)\right\} .
$$

Since $\mathcal{B}$ is linear, the above expression can be rearranged as an integral equation for $B_{e z}$, assuming that $\phi$ is known:

$$
\hat{\mathbf{e}}_{z} B_{e z}+a \sigma \mathcal{B}\left\{\hat{\mathbf{e}}_{y} v_{x} B_{e z}\right\}=a \sigma \mathcal{B}\left\{-\nabla_{t} \phi-\hat{\mathbf{e}}_{y} v_{x} B_{0 z}\right\}
$$

This equation can be numerically solved and the solution can be substituted to (9) which yields

$$
-\nabla \times \nabla_{t} \phi=v_{x} \frac{\partial B_{0 z}}{\partial y}+v_{x} \frac{\partial B_{e z}}{\partial y}
$$


As $B_{e z}$ depends on $\phi$, (13) can be solved by, e.g., FEM, combined with an iterative scheme implemented in the Matlab ${ }^{\circledR}$ pdenonlin function.

\subsection{Numerical evaluation of the Biot-Savart law on a finite element mesh}

The solution of the integral equation (12) is obtained within the frame of the applied mesh-discretisation of FEM. A finite surface $S$ in the $x y$ plane below the magnet is meshed; let $n$ be the number of nodes and $m$ be the number of triangles of the mesh respectively. Using the nodal values $\phi^{k}, B_{0 z}^{k}, B_{e z}^{k}(k=1,2, \ldots$, $n$ ), a piecewise constant approximation can be established for $\mathbf{K}$, based on (7). Let the current over the $l$-th triangle be denoted by $\mathbf{K}^{l}$, the barycentre coordinates of this triangle are $\left(\chi_{l}, \psi_{l}\right)$ and its area is $S_{l}$, respectively $(l=1,2, \ldots, m)$. The Biot-Savart law (8) is approximated and the reaction field at the $k$-th node $\left(x_{k}, y_{k}\right)$ is expressed as:

$$
\hat{\mathbf{e}}_{z} B_{e z}\left(x_{k}, y_{k}\right)=\frac{\mu_{0}}{4 \pi} \sum_{l=1}^{m} \frac{\mathbf{K}^{l} \times\left[\left(x_{k}-\chi_{l}\right) \hat{\mathbf{e}}_{x}+\left(y_{k}-\psi_{l}\right) \hat{\mathbf{e}}_{y}\right]}{\left[\left(x_{k}-\chi_{l}\right)^{2}+\left(y_{k}-\psi_{l}\right)^{2}\right]^{3 / 2}} S_{l},
$$

that is, a Riemann sum replaces the integral. By expressing $B_{e z}^{k}=B_{e z}\left(x_{k}, y_{k}\right)$ for all $k=1,2, \ldots, n$ using (14), and by enforcing (12) at all $n$ mesh nodes, a linear system of $n$ equations for the $n$ nodal values of the reaction field $B_{e z}^{k}$ is obtained. This is solved in each cycle of the iterative scheme of pdenonlin.

\subsection{Results for the moving plate}

In the numerical study, a copper plate with a conductivity of $\sigma=57 \mathrm{MS} / \mathrm{m}$ and thickness $a=1 \mathrm{~mm}$ is considered (note that neither $\sigma$, nor $a$ appears alone anywhere, but their product counts in this model). The distance of the magnetic dipole is $h=10 \mathrm{~cm}$. The model domain in the FEM computation is a half-circle with a radius $r=50 \mathrm{~cm}$. The diameter of the halfcircle lies along the $\mathrm{x}$ axis and the line-symmetry (to the $x$ axis) is taken into account in the implementation.

In Figure 4, the $y$ component of the surface current density is plotted along the $x$ axis. Let us recall that the "approximate" method involves the solution of (10), whereas the "proper" method is based on (13) and (14). In Figure 5, the distribution of the surface current density over the $x y$ plane is visualized by the current-paths.

\section{Conclusion}

The numerical calculation of the eddy-current distribution within conductors due to a moving magnetic dipole has been studied, in two standard demonstrative cases.

Special emphasis is put on that component of the total magnetic field which is generated by the induced eddy-currents. This component is much smaller than the imposed magnetic field in the standard configurations at small velocities, so it is

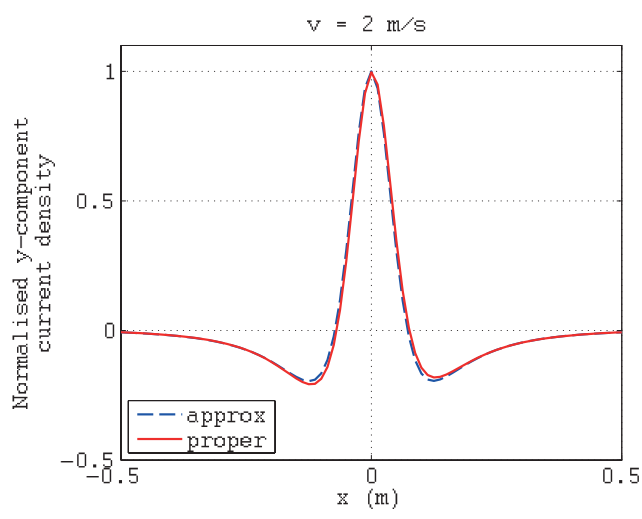

(a) Small speed, the difference is not significant.

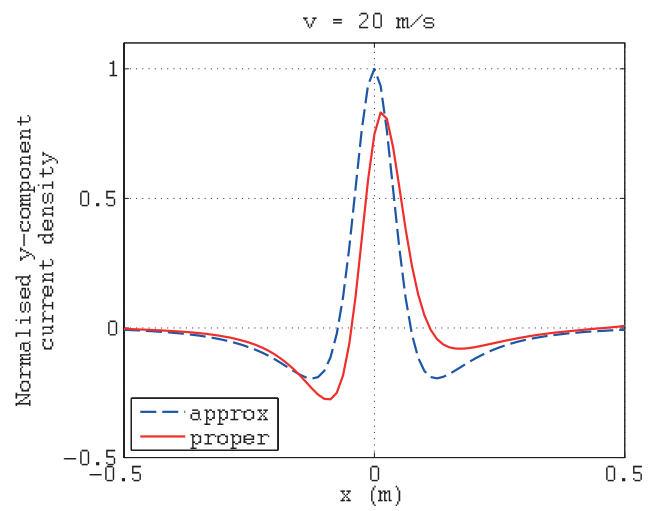

(b) As $v$ increases, the approximate calculation differs more and more from the proper results

Fig. 4 Plate-example: normalised $y$ component of the surface current density at different velocities, along the $x$ axis. With and without the contribution of the reaction field.

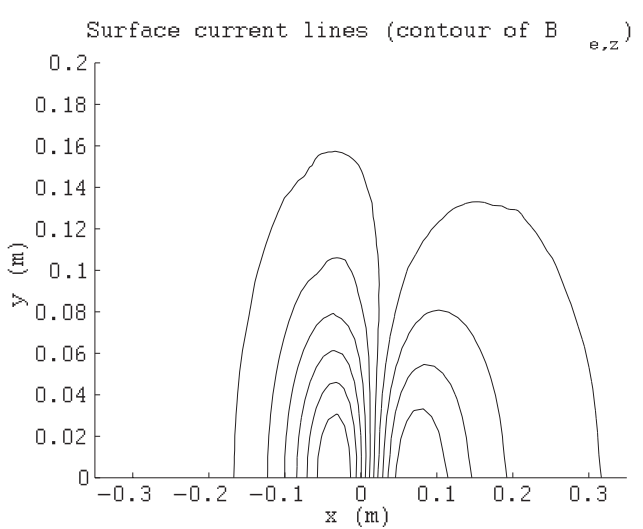

Fig. 5 Plate-example: paths of the surface current at $v_{x}=20 \mathrm{~m} / \mathrm{s}$. The current distribution is not symmetric to the $y$ axis due to the reaction effect of the induced current itself.

often neglected. However, we pointed out that it does have a considerable effect at higher velocities, and also emphasized that from an educational point of view, one should take it into account. A 2D FEM simulator has been used for the calculations, which are thus thought to be easy to follow and reproduce by university students. 


\section{Acknowledgements}

This work was supported by the Hungarian Scientific Research Fund under grants K-105996 and K-111987.

\section{References}

[1] Irvine, B., Kemnetz, M., Gangopadhyaya, A., Ruubel, T. "Magnet traveling through a conducting pipe: a variation on the analytical approach." American Journal of Physics. 82 (4). pp. 273-279. 2012. DOI: $10.1119 / 1.4864278$

[2] Donoso, G., Ladera, C. L., Martin, P. "Magnet fall inside a conductive pipe: motion and the role of the pipe wall thickness." European Journal of Physics. 30 (4). pp. 855-869. 2009. DOI: 10.1088/0143-0807/30/4/018

[3] Levin, Y., da Silveira, F. L., Rizzato, F. B. "Electromagnetic braking: A simple quantitative model." American Journal of Physics. 74 (9). 2006. pp. 815-817. DOI: 10.1119/1.2203645

[4] Bae, J-S., Hwang, J-H., Park, J-S., Kwag, D-G. "Modeling and experiments on eddy current damping caused by a permanent magnet in a conductive tube." Journal of Mechanical Science and Technology. 23 (11). pp. 3024-3035. 2009. DOI: 10.1007/s12206-009-0819-0

[5] Bonnano, A., Bozzo, G., Camarca, M., Sapia, P. "Using a PC and external media to quantitatively investigate electromagnetic induction." Physics Education. 46 (4). pp. 386-395. 2011. DOI: 10.1088/0031-9120/46/4/001
[6] Salzman, P. J., Burke, J. R., Lea, S. M. "The Effect of Electric Fields In A Classic Introductory Physics Treatment of Eddy Current Forces." American Journal of Physics. 69 (5). pp. 586-590. 2001. DOI: 10.1119/1.1341249

[7] Evgeny, V. V., Thess, A. "Interaction of a magnetic dipole with a slowly moving electrically conducting plate." Journal of Engineering Mathematics. 77 (1). pp. 147-161. 2012. DOI: 10.1007/s10665-012-9545-1

[8] Thess, A., Votyakov, E. V., Kolesnikov, Y. "Lorentz Force Velocimetry." Physical Review Letters. 96 (16). 2006. DOI: $10.1103 /$ physrevlett.96.164501

[9] Zec, M., Uhling, R. P., Ziolkowski, M., Brauer, H. "Fast Technique for Lorentz Force Calculations in Non-DestructiveTesting Applications." IEEE Transactions on Magnetics. 50 (2). pp. 133-136. 2014. DOI: 10.1109/TMAG.2013.2281971

[10] Smaill, C. R., Rowe, G. B., Godfrey, E., Paton, R. O. "An Investigation Into the Understanding and Skills of First-Year Electrical Engineering Students." IEEE Transactions on Education. 55 (1). pp. 29-35. 2012. DOI: 10.1109/TE.2011.2114663

[11] Cheng, D. K. "Field and wave electromagnetics." Addison-Wesley Publishing Company. 1983. 